RUNHETC-2002-47, UPR-1030-T

hep-th/0303208

\title{
Dynamical Supersymmetry Breaking in Standard-like Models with Intersecting D6-branes
}

\author{
Mirjam Cvetič ${ }^{1 *}$, Paul Langacker ${ }^{2}$, and Jing Wang ${ }^{3}$ \\ 1 Department of Physics and Astronomy, \\ Rutgers University, Piscataway, NJ 08855-0849, USA \\ and \\ School of Natural Science, Institute for Advanced Study, \\ Olden Lane, Princeton, NJ 08540, USA \\ 2 Department of Physics and Astronomy, University of Pennsylvania, Philadelphia, PA 19104 \\ 3 Fermilab, Theory Division, Batavia, IL 60510, USA
}

(November 3, 2018)

\begin{abstract}
We address dynamical supersymmetry breaking within a $N=1$ supersymmetric Standard-like Model based on a $Z_{2} \times Z_{2}$ Type IIA orientifold with intersecting D6-branes. The model possesses an additional, confining gauge sector with the $U S p(2)_{A} \times U S p(2)_{B} \times U S p(4)$ gauge group, where the gaugino condensation mechanism allows for the breaking of supersymmetry and stabilizes moduli. We derive the leading contribution to the non-perturbative effective superpotential and determine numerically the minima of the supergravity potential. These minima break supersymmetry and fix two undetermined moduli, which in turn completely specify the gauge couplings at the string scale. For this specific construction the minima have a negative cosmological constant. We expect that for other supersymmetric Standard-like models with intersecting D6-branes, which also possess confining gauge sectors, the supersymmetry breaking mechanism would have qualitatively similar features.
\end{abstract}

11.25.-W

Typeset using REVTEX

* On Sabbatic Leave from University of Pennsylvania. 


\section{INTRODUCTION}

The second string revolution and the advent of D-branes opened the door for the construction of open string solutions, which correspond to the strongly coupled heterotic string sector. The techniques of conformal field theory in describing D-branes and orientifold planes on orbifolds allow for the construction of consistent four-dimensional $N=1$ supersymmetric models based on Type II orientifolds. Particular models, represented in Refs. [1-12], are based on constructions with D-branes located at orbifold singularities, and chiral fermions appear on the world-volumes of the D-branes.

An alternative construction with chiral fermions, that has been explored only recently, is that of Type II orientifold models with intersecting branes. Chiral fermions appear in the open string spectrum, localized at the intersections [13]. The model-building with intersecting branes was developed [14-18] (and subsequently explored in [19-23]), where constructions of non-supersymmetric brane world models were primarily addressed. Numerous examples of non-supersymmetric three-family Standard-like models as well as GUT models were obtained. However, the stability of non-supersymmetric models is not well understood, especially when the string scale is close to the Planck scale, since the non-supersymmetric models are subject to large quantum corrections. Typically, the models are unstable when D-branes are intersecting at angles, since supersymmetry is generically broken.

On the other hand, examples of $\mathcal{N}=1$ supersymmetric orientifold models with branes at angles were constructed in [24-26], resulting in quasi-realistic models containing the threefamily Standard Model. An example of a supersymmetric $S U(5)$ GUT model with four families of quarks and leptons (i.e., a net number of four $\mathbf{1 0}$-plets and four $\overline{\mathbf{5}}$-plets) was also presented in [25]. The original construction is based on an $\mathbf{Z}_{2} \times \mathbf{Z}_{2}$ orientifold with D6branes wrapping specific supersymmetric three-cycles of the six-torus $\left(T^{6}=T^{2} \times T^{2} \times T^{2}\right)$.

Recently, a new example of the supersymmetric three-family left-right symmetric model based on an $\mathbf{T}^{\mathbf{6}} / \mathbf{Z}_{\mathbf{4}}$ orientifold was constructed [27]. Further developments [28] involve the construction of a larger class of supersymmetric three-family Standard-like Models, based on $\mathbf{T}^{\mathbf{6}} /\left(\mathbf{Z}_{\mathbf{2}} \times \mathbf{Z}_{\mathbf{2}}\right)$ orientifolds, by exploring the wrapping of D6-branes along more general supersymmetric three-cycles (and implementing RR tadpole cancellation conditions). A systematic exploration of a general class of supersymmetric three-family $S U(5)$ GUT models arising from $\mathbf{T}^{\mathbf{6}} /\left(\mathbf{Z}_{\mathbf{2}} \times \mathbf{Z}_{\mathbf{2}}\right)$ orientifolds with D6-branes wrapping general supersymmetric three-cycles was most recently presented in [29].

These quasi-realistic constructions provide a testing ground to further address the phenomenology of such constructions. ${ }^{1}$ A preliminary phenomenological study of the first three -family Standard-like model $[24,25]$ was explored in $[35,36]$.

In [35] a detailed study of the gauge couplings and their renormalization group (RG) flow

\footnotetext{
${ }^{1}$ These models [24-29] correspond in the strong coupling limit to compactifications of $\mathrm{M}$ theory on certain singular $G_{2}$ manifolds. As discussed in [26], the D-brane picture provides a description of how chiral fermions arise from singularities of $G_{2}$ compactifications [30-32,24,25]. Recently, there has been an exploration of phenomenological features (e.g., the problem of doublet-triplet splitting, threshold corrections, and proton decay) of GUT models derived from $G_{2}$ compactifications $[33,34]$. It would be interesting to explore related features in this class of orientifold models.
} 
were studied. At the string scale these couplings depend on an additional modulus parameter $\chi \equiv R_{2}^{1} / R_{1}^{1}$, where $R_{1,2}^{i}$ are the respective radii of the $i$-th two-torus. The Standard-Model gauge sector does not predict realistic low-energy values of gauge couplings (primarily due to the additional Higgs and exotic fields in the massless spectrum). On the other hand the additional non-Abelian gauge sector with the gauge group $U S p(2)_{A} \times U S p(2)_{B} \times S p(4)$ has negative values of the $\beta$ functions, and thus allows for a confining phase in the infra red regime. The gaugino condensation can in turn take place and trigger dynamical supersymmetry breaking there. Charge confinement also implies the interesting feature that the left-handed members of an exotic $(S U(2)$-singlet) family can become composite while their right-handed partners are elementary.

The main purpose of this note it to address dynamical supersymmetry breaking in the supersymmetric Standard-like model with intersecting D6-branes [24,25]. The approach is is based on the study of $N=1$ super Yang Mills (SYM) theory with a confining phase in the infra red regime. There the gaugino condensation generates a a non-perturbative effective superpotential [37]. A subsequent minimization of the supergravity potential in turn determines the ground state, which in certain cases breaks supersymmetry. (For recent exciting developments involving the exact non-perturbative superpotential, which includes all higher order instanton corrections, for large classes $N=1$ super Yang Mills theories, see $[38,39]$ and references therein.)

We shall show that the additional gauge sectors of the supersymmetric Standard-like Model allow for dynamical supersymmetry breaking via gaugino condensation. For the specific example we calculated the explicit dependence of the non-perturbative superpotential on the moduli fields $S$ (dilaton) and $U$ (complex structure modulus) of one of the three internal two-tori; the other two are fixed due to the supersymmetry constraint of the string construction. The minimization of the explicit supergravity potential in turn produces isolated, supersymmetry breaking minima, with both moduli $S$ and $U$ fixed. These moduli completely determine the values of the gauge couplings in the theory at the string scale. Unfortunately, the specific example has the property that the value of the potential at the minimimum is negative and of the order of the string scale.

While we address a specific model, we expect that the qualitative features would be generic in other models, with intersecting branes and confining gauge sectors, such as constructed in [28]. All of these examples typically have a number of non-Abelian confining gauge group factors, typically associated with USp groups. The non-perturbative superpotential, that is a sum of exponential factors that typically depend on the dilaton $S$ and complex structure moduli $U_{i}$, will allow for minima in which such moduli are stabilized.

The paper is organized as follows. We summarize in Section 2 the results for the gauge group couplings and the explicit dependence of the gauge coupling on moduli $S$ and $U_{i}$, first in a general case of models with intersecting branes, and then for the specific model considered. In Section 3 we determine the explicit form of the non-perturbative superpotential, due to the leading instanton contribution, as a function of moduli and then focus on the concrete example. We further minimize the supergravity potential numerically and analyse the features of the minima, including implications for the values of gauge couplings and gaugino masses. Conclusions are given in Section 4, where we contrast our results with those for the perturbative heterotic string constructions. 


\section{MODEL}

\section{A. Essential Features of the Model}

In this section we shall provide the key features of the construction. We refer the reader to the original papers $[24,25]$ for more detailed discussions.

For concreteness, we consider an orientifold of type IIA on $\mathbf{T}^{6} /\left(\mathbf{Z}_{2} \times \mathbf{Z}_{2}\right)$. The orbifold actions have generators $\theta, \omega$ acting as $\theta:\left(z_{1}, z_{2}, z_{3}\right) \rightarrow\left(-z_{1},-z_{2}, z_{3}\right)$, and $\omega:\left(z_{1}, z_{2}, z_{3}\right) \rightarrow$ $\left(z_{1},-z_{2},-z_{3}\right)$ on the complex coordinates $z_{i}$ of $\mathbf{T}^{6}$, which is assumed to be factorizable. The orientifold action is $\Omega R$, where $\Omega$ is world-sheet parity, and $R$ acts by $R:\left(z_{1}, z_{2}, z_{3}\right) \rightarrow$ $\left(\bar{z}_{1}, \bar{z}_{2}, \bar{z}_{3}\right)$. The model contains four kinds of O6-planes, associated to the actions of $\Omega R$, $\Omega R \theta, \Omega R \omega, \Omega R \theta \omega$. The cancellation of the $\mathrm{RR}$ crosscap tadpoles requires an introduction of $K$ stacks of $N_{a}$ D6-branes $(a=1, \ldots, K)$ wrapped on three-cycles (taken to be the product of 1-cycles $\left(n_{a}^{i}, m_{a}^{i}\right)$ in the $i^{t h}$ two-torus), and their images under $\Omega R$, wrapped on cycles $\left(n_{a}^{i},-m_{a}^{i}\right)$.

The cancellation of untwisted tadpoles imposes constraints on the number of D6-branes and the types of 3-cycles that they wrap around. The cancellation of twisted tadpoles determines the orbifold actions on the Chan-Paton indices of the branes (which are explicitly given in $[24,25])$.

The condition that the system of branes preserves $\mathcal{N}=1$ supersymmetry requires [13] that each stack of D6-branes is related to the O6-planes by a rotation in $S U(3)$ : denoting by $\theta_{i}$ the angles the D6-brane forms with the horizontal direction in the $i^{\text {th }}$ two-torus, supersymmetry preserving configurations must satisfy $\theta_{1}+\theta_{2}+\theta_{3}=0$. This in turn impose a constraint on the wrapping numbers and the complex structure moduli $\chi_{i}=R_{2}^{i} / R_{1}^{i}$.

The rules to compute the spectrum are analogous to those in [16]. We summarize the resulting chiral spectrum in Table I, found in [24,25], where

$$
I_{a b}=\left(n_{a}^{1} m_{b}^{1}-m_{a}^{1} n_{b}^{1}\right)\left(n_{a}^{2} m_{b}^{2}-m_{a}^{2} n_{b}^{2}\right)\left(n_{a}^{3} m_{b}^{3}-m_{a}^{3} n_{b}^{3}\right)
$$




\section{TABLES}

\begin{tabular}{|c|c|}
\hline \hline \hline Sector & Representation \\
\hline \hline \multirow{2}{*}{$a a$} & $U\left(N_{a} / 2\right)$ vector multiplet \\
& 3 Adj. chiral multiplets \\
\hline \hline$a b+b a$ & $I_{a b}$ chiral multiplets in $\left(N_{a} / 2, \overline{N_{b} / 2}\right)$ rep. \\
\hline \hline$a b^{\prime}+b^{\prime} a$ & $I_{a b^{\prime}}$ chiral multiplets in $\left(N_{a} / 2, N_{b} / 2\right)$ rep. \\
\hline \hline$a a^{\prime}+a^{\prime} a$ & $-\frac{1}{2}\left(I_{a a^{\prime}}-\frac{4}{2^{k}} I_{a, O 6}\right)$ chiral multiplets in sym. rep. of $U\left(N_{a} / 2\right)$ \\
& $-\frac{1}{2}\left(I_{a a^{\prime}}+\frac{4}{2^{k}} I_{a, O 6}\right)$ chiral multiplets in antisym. rep. of $U\left(N_{a} / 2\right)$ \\
\hline \hline
\end{tabular}

TABLE I. General spectrum on D6-branes at generic angles (namely, not parallel to any O6-plane in all three tori). The spectrum is valid for tilted tori. The models may contain additional non-chiral pieces in the $a a^{\prime}$ sector and in $a b$, $a b^{\prime}$ sectors with zero intersection, if the relevant branes overlap.

The D6-brane configuration for the first example leading to a three-family Standardlike Model is provided in table II, and satisfies the tadpole cancellation conditions. The configuration is supersymmetric for

$$
\chi_{1}: \chi_{2}: \chi_{3}=1: 3: 2 .
$$

The weak hypercharge is given by

$$
Y=(B-L) / 2+\left(Q_{8}+Q_{8^{\prime}}\right) / 2
$$

where $B-L=Q_{3} / 3-Q_{1}$ and $Q_{3}$ is the charge corresponding to the $U(1)$ in $U(3)_{C}$.

\begin{tabular}{|c|c|c|c|}
\hline \hline Type & Gauge Group & $N_{a}$ & $\left(n_{a}^{1}, m_{a}^{1}\right) \times\left(n_{a}^{2}, m_{a}^{2}\right) \times\left(n_{a}^{3}, \widetilde{m}_{a}^{3}\right)$ \\
\hline$A_{1}$ & $U S p(8) \rightarrow U(1)_{8} \times U(1)_{8^{\prime}}$ & 8 & $(0,1) \times(0,-1) \times(2, \widetilde{0})$ \\
$A_{2}$ & $U S p(2)_{A}$ & 2 & $(1,0) \times(1,0) \times(2, \widetilde{0})$ \\
\hline$B_{1}$ & $U(2)_{L}$ & 4 & $(1,0) \times(1,-1) \times(1, \widetilde{3 / 2})$ \\
$B_{2}$ & $U S p(2)_{B}$ & 2 & $(1,0) \times(0,1) \times(0, \widetilde{-1})$ \\
\hline$C_{1}$ & $U(4) \rightarrow U(3)_{C} \times U(1)_{1}$ & $6+2$ & $(1,-1) \times(1,0) \times(1, \widetilde{1 / 2})$ \\
$C_{2}$ & $U S p(4)$ & 4 & $(0,1) \times(1,0) \times(0, \widetilde{-1})$ \\
\hline \hline
\end{tabular}

TABLE II. D6-brane configuration for the three-family model.

The resulting spectrum is given in the original paper $[24,25]$ and the subsequent papers $[35,36]$. 


\section{B. Gauge Couplings}

We shall summarize the results of the gauge coupling calculations for the model. Since the gauge couplings are associated with different stacks of branes they do not exhibit a conventional gauge unification. Nevertheless, the value of each gauge coupling at the string scale is predicted in terms of a modulus $\chi$ and the ratio of the Planck to string scales. The running is strongly affected by the exotic matter and multiple Higgs fields, leading to low values of the MSSM sector couplings at low energy. However, the hidden sector groups are asymptotically free.

The gauge coupling of the gauge field from a stack of $D 6$-branes wrapping a three-cycle is given by

$$
\frac{1}{g_{Y M}^{2}}=\frac{M_{s}^{3} V_{3}}{(2 \pi)^{4} g_{s}}
$$

where $M_{s}=1 / \sqrt{\alpha^{\prime}}$ is the string scale, $g_{s}$ is the string coupling and $V_{3}$ is the volume of the three-cycle wrapped by a particular D6-brane. For our specific cases $V_{3}$ is given by ${ }^{2}$ :

$$
V_{3}=\frac{\mathbf{1}}{\mathbf{4}}(2 \pi)^{3} \prod_{i=1}^{3} \sqrt{n^{i 2}\left(R_{1}^{i}\right)^{2}+\hat{m}^{i 2}\left(R_{2}^{i}\right)^{2}},
$$

where $R_{1,2}^{i}$ are the radii of the two dimensions of the $i^{\text {th }}$ two-torus, $\hat{m}^{i}=m^{i}$ for $i=1,2$, $\hat{m}^{3}=\tilde{m}^{3}=m^{3}-\frac{1}{2} n^{3}$ (the third $T^{2}$ is tilted), and the wrapping numbers $\left(n^{i}, \hat{m}^{i}\right)$ are given in Table II. One can trade $g_{s}$ in (II.4) for the four-dimensional Planck-scale $M_{P}^{(4 d)}$ which is defined as the coefficient of the Einstein term in the low energy effective action:

$$
S_{4 d}=\left(M_{P}^{(4 d)}\right)^{2} \int d x^{4} \sqrt{g} R+\ldots=\frac{1}{16 \pi G_{N}} \int d x^{4} \sqrt{g} R+\ldots
$$

Since $G_{N}^{-1 / 2}=1.22 \times 10^{19} \mathrm{GeV}$, we have $M_{P}^{(4 d)}=\frac{1}{4 \sqrt{\pi}} \times G_{N}^{-1 / 2}=1.7 \times 10^{18} \mathrm{GeV}$. The Planck scale is related to the string coupling $g_{s}$ and string scale $M_{s}$ by

$$
\left(M_{P}^{(4 d)}\right)^{2}=\frac{M_{s}^{8} V_{6}}{(2 \pi)^{7} g_{s}^{2}}
$$

where $V_{6}$ is the total internal volume given by

$$
V_{6}=\frac{(2 \pi)^{6}}{4} \prod_{i=1}^{3} R_{1}^{i} R_{2}^{i}
$$

\footnotetext{
${ }^{2}$ The definition of $V_{3}$ in (II.5) differs from one in [35] by a factor of $\frac{1}{4}$. This factor has to be included, due to the orbifolding of $T^{6}$ by $\mathbf{Z}_{\mathbf{2}} \times \mathbf{Z}_{\mathbf{2}}$, which is an Abelian group of order 4 . This implies that the expressions in [35] for $g_{Y M}^{2}$ and $\alpha_{G}$ should be increased by a factor of 4 . The numerical results in [35], which were given for $M_{s} \sim M_{P}^{(4 d)}$, are still approximately valid for the case $M_{s} \sim M_{P}^{(4 d)} / 4$.
} 
Again, the factor of $\frac{1}{4}$ is due to the orbifolding of $T^{6}$ by $\mathbf{Z}_{2} \times \mathbf{Z}_{2}$. This factor was included in [35]. Employing (II.7) allows us to write the gauge couplings in terms of $M_{s}, M_{P}^{(4 d)}, V_{3}$ and $V_{6}$ :

$$
g_{Y M}^{2}=\sqrt{2 \pi} \frac{M_{s} \sqrt{V_{6}}}{M_{P}^{(4 d)} V_{3}}
$$

which in terms of the complex structure moduli $\chi_{i}=R_{2}^{i} / R_{1}^{i}$ becomes

$$
g_{Y M}^{2}=\frac{\sqrt{8 \pi} M_{s}}{M_{P}^{(4 d)}} \frac{\sqrt{\chi_{1} \chi_{2} \chi_{3}}}{\prod_{i=1}^{3} \sqrt{n^{i 2}+\hat{m}^{i 2} \chi_{i}^{2}}} .
$$

The supersymmetric condition (II.2) implies

$$
g_{Y M}^{2}=\frac{4 \sqrt{3 \pi} M_{s}}{M_{P}^{(4 d)}} \cdot \frac{\chi^{3 / 2}}{\sqrt{\left[\left(n^{1}\right)^{2}+\left(m^{1}\right)^{2} \chi^{2}\right]\left[\left(n^{2}\right)^{2}+9\left(m^{2}\right)^{2} \chi^{2}\right]\left[\left(n^{3}\right)^{2}+4\left(\tilde{m}^{3}\right)^{2} \chi^{2}\right]}},
$$

where $\chi \equiv \chi_{1}$.

At a scale $M$ below the string scale, the coupling $\alpha_{a}=\frac{g_{a}^{2}}{4 \pi}$ of the $a^{\text {th }}$ gauge factor is given (at one loop) by

$$
\frac{1}{\alpha_{a}(M)}=\frac{c_{a}(\chi)}{\alpha_{G}(\chi)}+\beta_{a} t
$$

where

$$
\alpha_{G}(\chi)=\sqrt{\frac{3}{\pi}} \frac{M_{s}}{M_{P}^{(4 d)}} \chi^{3 / 2},
$$

and

$$
t=\frac{1}{2 \pi} \ln \frac{M_{s}}{M} .
$$

The low energy predictions for the model are given in [35] (There, after correcting for the factor $1 / 4$ in (II.5), $M_{s} \sim M_{P}^{(4 d)} / 4$ was assumed, and the low energy result depends on one modulus parameter $\chi$.) Since we focus on the additional confining gauge sector we state the values of $c_{a}$ and $\beta_{a}$ for these gauge couplings in Table III. In [35] the renormalization group equations were studied without the inclusion of the chiral supermultiplets associated with the open string sector of the brane. There are three copies of such states in the adjoint representation; they are due to the fact that the supersymmetric cycles wrapped by D6branes are not rigid. In the Standard-model sector they affect in a negative way the low energy predictions for the standard model gauge couplings. However, in the quasi-hidden sector the only such states are associated with the $U S p(4)$ gauge group, where they change the beta function there from -5 to -2 . For the sake of completeness we include them in the study of gaugino condensation. 


\begin{tabular}{|c||c|c|}
\hline \hline Group $a$ & $c_{a}$ & $\beta_{a}$ (int) \\
\hline$U S p(2)_{B}$ & $6 \chi^{2}$ & -4 \\
$U S p(2)_{A}$ & 2 & -6 \\
$U S p(4)$ & $2 \chi^{2}$ & $-2(-5)$ \\
\hline \hline
\end{tabular}

TABLE III. Coefficients $c_{a}$ of $1 / \alpha_{G}$, and $\beta$ functions $\beta_{a}$ for the $U S p(2)_{B}$ and $U S p(2)_{A}$, associated with the $B_{2}$ and $A_{2}$ brane configuration, respectively, and $U S p(4)$ group associated with the $C_{2}$ brane configuration. The beta function of $-2(-5)$ for $U S p(4)$ includes (does not include) the contributions of three chiral 5 -plets that are not localized at intersections. 
In the following subsection we shall derive the explicit complex moduli dependence of the gauge couplings, which are suitable for the determination of the effective non-perturbative superpotential.

\section{Gauge Kinetic Function and Kähler Potential in Terms of Complex Structure Moduli}

To determine the moduli dependence of the gauge couplings in Type IIA theory with D6 branes in terms of complex structure moduli, we shall employ the fact that Type IIA theory with D6 branes is T-dual to Type I theory with D9 branes and background B-fluxes. Hence, to arrive at the proper definition of the moduli fields we shall start by writing down the moduli fields in the Type I theory with D9 branes, which are well known (see for example [40]). We then apply the duality transformations to arrive at the moduli fields for D6 branes.

In the Type I string with D9 branes, the real part of the dilaton $S$ has the familiar expression:

$$
R e(S)=\frac{M_{s}^{6} \Pi_{i=1}^{3} R_{1}^{i} R_{2}^{i}}{2 \pi g_{s}}
$$

The real part of the Kähler moduli $T^{i}$ are defined as

$$
R e\left(T^{i}\right)=\frac{M_{s}^{2} R_{1}^{i} R_{2}^{i}}{2 \pi g_{s}} .
$$

Again, $R_{1,2}^{i}$ are the radii of the of the $i$-th torus, $M_{s}=\frac{1}{\alpha^{\prime}}$ is the string scale $\left(\alpha^{\prime}\right.$ is the string tension) and $g_{s}$ is the string coupling.

The T-duality transformations between D9 branes with B-fluxes and D6 branes wrapped on 3-cycles are the following (see e.g., [40]),

$$
\begin{gathered}
R_{2}^{i} \rightarrow \frac{1}{M_{s}^{2} R_{2}^{i}}, \\
R_{1}^{i} \rightarrow R_{1}^{i}, \\
g_{s} \rightarrow \frac{g_{s}}{M_{s}^{3} \Pi_{i=1}^{3} R_{2}^{i}} .
\end{gathered}
$$

Under these transformations, the real part of the dilaton $S$ and the complex structure moduli $U^{i}$ take the following form:

$$
\begin{aligned}
R e(S) & =\frac{M_{s}^{3} \Pi_{i=1}^{3} R_{1}^{i}}{2 \pi g_{s}}, \\
R e\left(U^{i}\right) & =\frac{M_{s}^{3} R_{1}^{i} R_{2}^{j} R_{2}^{k}}{2 \pi g_{s}},
\end{aligned}
$$


where $i \neq j \neq k$.

The expression for $g_{Y M}^{2}$ (II.4) is determined in terms of $V_{3}$ (II.5). The supersymmetry constraint for the particular model requires the condition (II.2) on the $\chi_{i}=\frac{R_{2}^{i}}{R_{1}^{i}}$. It turns out [40] that these relations ensure that the volume of the 3-cycle $V_{3}$ in (II.5) can be written:

$$
\begin{aligned}
V_{3} & =\frac{\mathbf{1}}{4}(2 \pi)^{3}\left(n^{1} n^{2} n^{3} R_{1}^{(1)} R_{1}^{(2)} R_{1}^{(3)}-n^{1} \hat{m}^{2} \hat{m}^{3} R_{1}^{(1)} R_{2}^{(2)} R_{2}^{(3)}\right. \\
& \left.-\hat{m}^{1} n^{2} \hat{m}^{3} R_{2}^{(1)} R_{1}^{(2)} R_{2}^{(3)}-\hat{m}^{1} \hat{m}^{2} n^{3} R_{2}^{(1)} R_{2}^{(2)} R_{1}^{(3)}\right),
\end{aligned}
$$

where for the specific model $\hat{m}^{i}=m^{i}(i=1,2), \hat{m}^{3}=\tilde{m}^{3}=m^{3}-\frac{1}{2} n^{3}$. (It can be verified explicitly for each set of $\left(n^{i}, \hat{m}^{i}\right)$ in the model that eqs. (II.5) and (II.22) are indeed equivalent.)

Due to supersymmetry $g_{Y M}^{-2} \equiv R e(f)$, where the gauge kinetic function $f$ is a holomorphic function of the moduli $S$ and $U^{i}$. Given the above definition of the real part of the dilaton $S$ and the $U^{i}$ moduli and the form of $g_{Y M}^{-2}$ (II.4), with the $V_{3}$ derived in (II.22) one obtains:

$$
f=\frac{\mathbf{1}}{\mathbf{4}}\left[n^{1} n^{2} n^{3} S-n^{1} \hat{m}^{2} \hat{m}^{3} U^{1}-\hat{m}^{1} n^{2} \hat{m}^{3} U^{2}-\hat{m}^{1} \hat{m}^{2} n^{3} U^{3}\right] .
$$

It is indeed a holomorphic (and linear) function of the fields, as required by supersymmetry.

For the specific case of the additional (quasi-hidden)gauge sector,

$$
\begin{gathered}
f_{U S p(4)}=\frac{1}{4} U^{2}=\frac{1}{12} U, \\
f_{U S p(2)_{B}}=\frac{1}{4} U^{1}=\frac{1}{4} U, \\
f_{U S p(2)_{A}}=\frac{1}{2} S,
\end{gathered}
$$

where the second equality in the above equations follows from (II.2), which implies:

$$
U^{1}: U^{2}: U^{3}=1: \frac{1}{3}: \frac{1}{2} .
$$

and $U \equiv U^{1}$.

For the sake of completeness we also quote the gauge kinetic functions for the Standard Model sector:

$$
\begin{gathered}
f_{\left[U(3)_{C}, U(1)_{1}\right]}=\frac{1}{4}\left(S+\frac{1}{2} U^{2}\right)=\frac{1}{4}\left(S+\frac{1}{6} U\right), \\
f_{U(2)_{L}}=\frac{1}{4}\left(S+\frac{3}{2} U^{1}\right)=\frac{1}{4}\left(S+\frac{3}{2} U\right), \\
f_{\left[U(1)_{8}, U(1)_{8^{\prime}}\right]}=\frac{1}{2} U^{3}=\frac{1}{4} U .
\end{gathered}
$$

From (II.28), (II.30), and (II.3), one finds

$$
f_{Y}=\frac{5}{72}\left(S+\frac{59}{30} U\right)
$$

for weak hypercharge.

The Kähler potential for the fields is the so called no-scale potential. It takes the following canonical form:

$$
K=-\log (S+\bar{S})-\sum_{I=1}^{3} \log \left(U^{I}+\bar{U}^{I}\right)=-\log (S+\bar{S})-3 \log (U+\bar{U})+\log (6) .
$$

In (II.32) and elsewhere we have set the Planck scale $M_{P}^{(4 d)}$ to unity, i.e., all dimensional quantities are scaled by appropriate powers of $M_{P}^{(4 d)}$. 


\section{GAUGINO CONDENSATE AND EFFECTIVE MODULI POTENTIAL}

In local supersymmetric theory the gaugino condensate $\left\langle\lambda^{\alpha} \lambda_{\alpha}\right\rangle$ is contained in the definition of a chiral superfield:

$$
\mathcal{U} \equiv \mathcal{W}^{\alpha} \mathcal{W}_{\alpha}
$$

where $\mathcal{W}_{\alpha}$ is the vector superfield whose fermionic component is $\lambda^{\alpha}$. The confinement scale $\mu$ of the strongly coupled theory is defined as the scale at which the effective gauge coupling becomes strong and perturbation theory breaks down. Consequently, the gaugino condensate $\left|\left\langle\lambda^{\alpha} \lambda_{\alpha}\right\rangle\right| \propto \mu^{3}$. This can be generated by an exact effective superpotential for the chiral field $\mathcal{U}[37]$,

$$
W(\mathcal{U}, \Phi)=\frac{1}{4} \mathcal{U} f_{W}(\Phi)-\frac{\mathcal{U}}{32 \pi^{2}}\left(\beta \log \frac{\mathcal{U}}{\Lambda^{3}}+\text { const }\right)
$$

where $\Phi$ is the modulus field in the theory which determines the strength of the gauge coupling constants through the gauge kinetic functions $f_{W} ; \beta$ is the $\beta$-function coefficient of the strongly coupled group, and $\Lambda$ is the cut off scale of the theory.

The effective potential generates a VEV for $\mathcal{U}$,

$$
\mathcal{U}=\Lambda^{3} \exp \left(\frac{8 \pi^{2}}{\beta} f_{W}(\Phi)\right) \times \text { const. }
$$

Integrating out the field $\mathcal{U}$, an effective potential of the moduli fields can be generated,

$$
W_{e f f}(\Phi)=\frac{\beta}{32 \pi^{2}} \frac{\Lambda^{3}}{e} \exp \left(\frac{8 \pi^{2}}{\beta} f_{W}(\Phi)\right) \equiv d \Lambda^{3} \exp \left(b f_{W}(\Phi)\right)
$$

where we have defined the constants $d \equiv \frac{\beta}{32 e \pi^{2}}$ and $b \equiv \frac{8 \pi^{2}}{\beta}$.

The three groups $U S p(4), U S p(2)_{A}$, and $U S p(2)_{B}$ that become strongly coupled have the beta function coefficients $\beta_{1}=-2, \beta_{2}=-4$ and $\beta_{3}=-6$, respectively. With the previously defined gauge kinetic functions, the effective potential for the moduli fields $U$ and $S$ is

$$
W(U, S)=d_{1} \Lambda^{3} \exp \left(\frac{b_{1}}{12} U\right)+d_{2} \Lambda^{3} \exp \left(\frac{b_{2}}{4} U\right)+d_{3} \Lambda^{3} \exp \left(\frac{b_{3}}{2} S\right)
$$

where $d_{i}=\frac{\beta_{i}}{32 e \pi^{2}}$ and $b_{i}=\frac{8 \pi^{2}}{\beta_{i}}$. This is of course only the leading instanton contribution to the non-perturbative superpotential. It can be justified post-factum if the negative exponents are large at the minimum of the potential. This indeed turns out to be the case for the specific solution discussed in the next Subsection.

\section{A. Scalar Potential and its Ground States}

Given the Kähler potential and the effective superpotential of the moduli fields $U$ and $S$, one can derive the scalar potential for the moduli fields,

$$
V=\frac{1}{(S+\bar{S})(U+\bar{U})^{3}}\left\{\left|(S+\bar{S}) \frac{\partial W}{\partial S}-W\right|^{2}+3 \mid\left(\frac{U+\bar{U})}{3} \frac{\partial W}{\partial U}-\left.W\right|^{2}-3|W|^{2}\right\} .\right.
$$


In the above potential we have absorbed the coefficient $\sqrt{6}$ from the $\log 6$ term in the Kähler potential (II.32) in the definition of $\Lambda^{3} \rightarrow \sqrt{6} \Lambda^{3}$.

It is expected that the gauge coupling threshold corrections would introduce corrections that depend on toroidal Kähler moduli $T_{i}$ (see [41]) of the form that would modify the superpotential in a multiplicative way, i.e., $W_{\text {total }}=W_{0}\left(T_{i}\right) W(S, U)$, where $W_{0}$ typically depends on a product of Dedekind modular functions $\eta\left(T_{i}\right)$, i.e., $W_{0} \sim \prod_{i=1}^{3} \eta\left(T_{i}\right)^{-2}$. A superpotential contribution of that type, along with the Kähler potential $K=-\prod_{i=1}^{3} \log \left(T_{i}+\bar{T}_{i}\right)$, could in turn also contribute to supersymmetry breaking and stabilization of Kähler moduli. In this paper we are not including these effects, i.e., we assume that the dominant effects associated with the supersymmetry breaking come from the tree level gauge coupling contribution and are thus associated with the $S$ and $U$ sector contributions. We hope to return to the threshold correction contributions to the effective superpotential in the future.

It is difficult to derive an analytical expressions for the minimum of the potential, so we proceed with a numerical analysis.

The potential is periodic in $\operatorname{Im}(U)$ and $\operatorname{Im}(S)$, with periods $12 N / \pi$ and $3 M / \pi$, respectively, where $M$ and $N$ are integers. Thus, one can focus on finding the value of $\operatorname{Im}(U)$ and $\operatorname{Im}(S)$ in the "fundamental domain" $\{0,12 / \pi\}$ and $\{0,3 / \pi\}$, respectively. The numerical minimization yields the minimum at:

$$
\operatorname{Re}(S)=1.10, \quad \operatorname{Re}(U)=0.575, \quad \operatorname{Im}(S)=0.48+3 M / \pi, \quad \operatorname{Im}(U)=1.91+12 N / \pi
$$

Fig. 1 depicts the potential near the minimum as a function of moduli $S$ and $U$.

The value of the potential is negative at the minimum and is approximately $-3.5610^{-3} L^{2}$, where $L \equiv \sqrt{6} \Lambda^{3} /\left(32 \pi^{2} e\right)$. In the potential (III.38) we have set the Planck scale to 1 . The string scale is typically chosen to be of the same order as the Planck scale and thus $\Lambda=\mathcal{O}(1)$ (in Planck units). In our specific case (see the following subsection) $M_{s} \sim 1.85 M_{P}^{(4 d)}$ and thus $\Lambda^{3} \sim 6.33\left[M_{P}^{(4 d)}\right]^{3}$. As a consequence $L \sim 1.8110^{-2}\left[M_{P}^{(4 d)}\right]^{2}$, and the cosmological constant $\sim-1.16 \times 10^{-6}\left[M_{P}^{(4 d)}\right]^{4}$. Since all the other parameters of the potential at the minima are fixed, the large negative cosmological constant is inevitable.

The terms that dynamically break supersymmetry are significantly smaller than the contribution from the $-3|W|^{2}$ term. In particular:

$$
\begin{gathered}
F_{S} K^{S \bar{S}} \bar{F}_{\bar{S}}=\left(|(S+\bar{S}) \partial W / \partial S-W|^{2} /\left[(S+\bar{S})(U+\bar{U})^{3}\right] \sim 4.9510^{-7} L^{2}\right. \\
F_{U} K^{U \bar{U}} \bar{F}_{\bar{U}}=3\left|\frac{(U+\bar{U})}{3} \partial W / \partial U-W\right|^{2} /\left[(S+\bar{S})(U+\bar{U})^{3}\right] \sim 1.0310^{-7} L^{2} \\
-3|W|^{2} /[(S+\bar{S})(U+\bar{U})]^{3} \sim-3.5610^{-3} L^{2}
\end{gathered}
$$

Here $F_{\phi} \equiv e^{\frac{K}{2}}\left(\partial_{\phi} W+K_{\phi} W\right)$ and $K_{\phi} \equiv \partial_{\phi} K$.

In the case in which one does not include the matter contribution that is associated with the open string sector of the $U S p(4)$ brane, its beta function changes from -2 to -5 . In the latter case we found unstable points where $V \rightarrow-\infty$ as $\operatorname{Re}(U) \rightarrow 0$. One such point corresponds to $\operatorname{Im}(U)=4.242, \operatorname{Re}(S)=0.606, \operatorname{Im}(S)=0.388$ This phenomenon is due to the fact that in this case the negative contribution of the potential (arising from 
$-3|W|^{2}$ term) turns out to be dominant for small values of $\operatorname{Re}(U)$. We do not encounter this instability in the case $\beta=-2$, for which the relative strengths of the exponents in the effective superpotential balance in a way that the negative contributions to the potential do not dominate for small values of $\operatorname{Re}(U)$ and/or $\operatorname{Re}(S)$. 


\section{FIGURES}

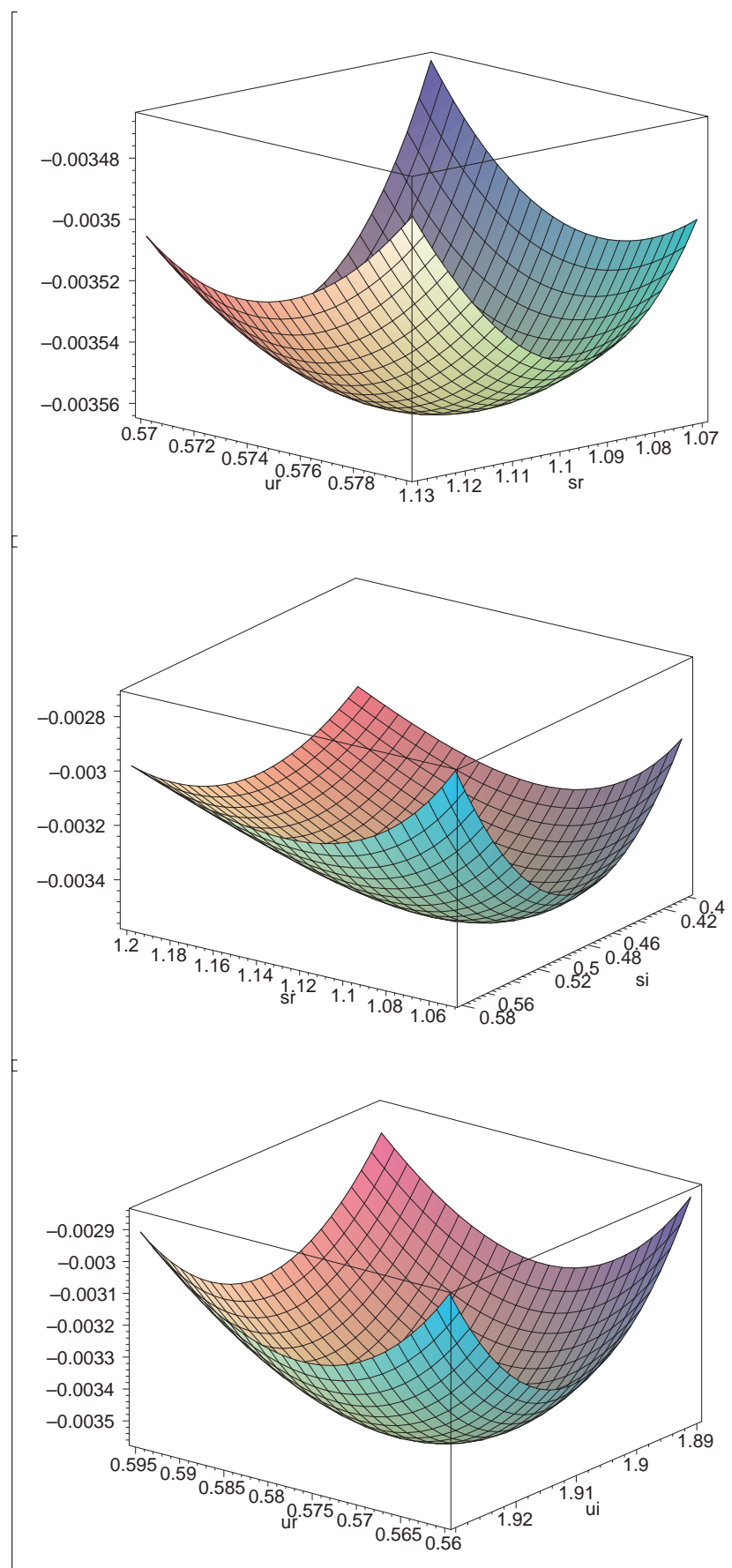

FIG. 1. Plots of potential $V$ (in units of $\Lambda^{2} \equiv\left[\sqrt{6} \Lambda^{3} /\left(32 \pi^{2} e\right)\right]^{2}$ ) as a function of $\operatorname{Re}(S)$ and $\operatorname{Re}(U)(\operatorname{Im}(U)=1.91$ and $\operatorname{Im}(S)=0.48), \operatorname{Re}(S)$ and $\operatorname{Im}(S)(\operatorname{Re}(U)=0.575$ and $\operatorname{Im}(U)=1.91)$, and $\operatorname{Re}(U)$ and $\operatorname{Im}(U)(\operatorname{Re}(S)=1.10$ and $\operatorname{Im}(S)=0.48)$, respectively. In the Figures $\operatorname{Re}(S), \operatorname{Im}(S), \operatorname{Re}(U)$, and $\operatorname{Im}(U)$ are denoted $s r$, si, ur, ui, respectively. 


\section{B. Phenomenology}

We first comment on features of the gauge couplings. The quantities $\chi$ and $1 / \alpha_{G}$ defined in (II.12) and (II.13) are related to the moduli by

$$
\chi=\sqrt{\frac{\operatorname{Re}(U)}{6 R e(S)}}, \quad \frac{1}{\alpha_{G}}=\pi R e(S) .
$$

At the minimum of the potential they take the values $\chi=0.295$ and $1 / \alpha_{G}=3.46$, corresponding to $M_{P}^{(4 d)} / M_{s}=0.542$, which satisfies the perturbative consistency condition $M_{P}^{(4 d)} / M_{s}>1 / \sqrt{8} \pi[35]$. From these values, and the expressions for the MSSM gauge parameters $c_{a}$ and $\beta_{a}$ given in Table VI of [35], we can calculate the predicted values of the standard model gauge couplings at the electroweak scale ${ }^{3}$. The inverse strong and electromagnetic couplings are predicted to be

$$
\frac{1}{\alpha_{3}}=52.2, \quad \frac{1}{\alpha}=525
$$

which are much larger than the respective experimental values $\sim 8.5$ and 128 . The unrealistically small values predicted for the gauge couplings are due to the extra chiral matter in the construction ${ }^{4}$. The weak angle $\sin ^{2} \theta_{W}$, which is a ratio of gauge couplings, fares somewhat better: it is predicted to be 0.29 , not too far from the experimental 0.23 .

Unfortunately, since the minima have negative cosmological constants, these vacua do not provide realistic backgrounds for a detailed study of the soft supersymmetry breaking parameters of the charged matter sector of the model. We defer this investigation for the future.

We can however determine gaugino masses in terms of the $F_{S}$ and $F_{U}$. The general expression for the gaugino mass (i.e., terms of the type $\lambda_{a} \lambda_{a}$ in the Lagrangian), is

$$
m_{\lambda_{a}}=\left(\partial_{\phi_{i}} f_{a}\right) K^{\phi_{i}} \bar{\phi}_{j} \bar{F}_{\bar{\phi}_{j}}
$$

Here $K^{\phi_{i} \bar{\phi}_{j}}$ is the inverse of the Kähler metric, $f_{a}$ is a gauge kinetic function, and $F_{\phi}$ was defined after Eq. (III.42) . In the Standard Model sector the gauge functions, determined in (II.28-II.30), yield the following expressions for gaugino masses at the string scale:

$$
\begin{aligned}
m_{\left[U(3)_{C}, U(1)_{1}\right]} & =\frac{1}{4} K^{S \bar{S}_{\bar{F}}} \overline{\bar{S}}+\frac{1}{24} K^{U \bar{U}} \bar{F}_{\bar{U}} \\
& =(1.89-3.48 I) 10^{-4} L \sim(3.42-6.30 I) 10^{-6} M_{P}^{(4 d)},
\end{aligned}
$$

\footnotetext{
${ }^{3} \mathrm{~A}$ fully realistic construction would predict the electroweak scale from the soft supersymmetry breaking. In our case, we simply use the experimental electroweak scale, which corresponds to $t=6.06$ in (II.14).

${ }^{4}$ Unlike in [35], we are also including the chiral states that are not localized at the brane intersections for consistency with our treatment of the strongly coupled confining sector. With these states, the strong $S U(3)$ group is not asymptotically free.
} 


$$
\begin{aligned}
& m_{U(2)_{L}}=\frac{1}{4} K^{S} \bar{S} \bar{F}_{\bar{S}}+\frac{3}{8} K^{U} \bar{U} \bar{F}_{\bar{U}}=(2.41-3.95 I) 10^{-4} L \sim(4.36-7.15 I) 10^{-6} M_{P}^{(4 d)}, \\
& m_{\left[U(1)_{8}, U(1)_{\left.8^{\prime}\right]}\right.}=\frac{1}{4} K^{U} \bar{U} \bar{F}_{\bar{U}}=(3.93-3.56 I) 10^{-5} L \sim(7.11-6.44 I) 10^{-7} M_{P}^{(4 d)},
\end{aligned}
$$

where we have restored the appropriate factor of $M_{P}^{(4 d)}$ in the final expressions. When a set of $U(1)^{\prime}$ 's with charges $Q_{a}$ is broken to a single $U(1)^{\prime}$ with charge $Q^{\prime}=\sum_{a} d_{a} Q_{a}$, then the $U(1)^{\prime}$ coupling and gaugino masses are related to those of the original factors by

$$
\frac{1}{\alpha^{\prime}}=\sum_{a} \frac{d_{a}^{2}}{\alpha_{a}}, \quad m^{\prime}=\frac{\sum_{a} \frac{d_{a}^{2}}{\alpha_{a}} m_{a}}{\sum_{a} \frac{d_{a}^{2}}{\alpha_{a}}} .
$$

From (II.3) one then obtains ${ }^{5}$

$$
m_{Y}=(1.20-2.03 I) 10^{-4} L \sim(2.17-3.67 I) 10^{-6} M_{P}^{(4 d)}
$$

These masses are non-universal, complex (indicating significant $C P$-violating phases), and the values for the specific solution are too large. As in all such constructions, the gaugino masses below the string scale satisfy the same RG equations at one loop as the corresponding gauge couplings, so that $m_{a}(t) / m_{a}(0)=\alpha_{a}(t) / \alpha_{a}(0)$. However, unlike heterotic constructions and simple grand unified theories, the gaugino masses and gauge couplings at the string scale depend on two moduli $S$ and $U$. These dependences are non-universal and are different for the gaugino masses and gauge couplings. Thus the gaugino unification prediction $m_{b}(t) / m_{a}(t)=\alpha_{b}(t) / \alpha_{a}(t)$ of those models is lost. Rather, one has

$$
\frac{m_{b}(t) \alpha_{a}(t)}{m_{a}(t) \alpha_{b}(t)}=\frac{m_{b}(0) f_{b}}{m_{a}(0) f_{a}}
$$

For example, for the minimum of the potential in this model, the right hand side of (III.51) is $0.52-0.026 I$ for $(b, a)=(S U(3), S U(2))$ and $10.6+0.16 I$ for $(b, a)=\left(S U(2), \sqrt{\frac{5}{3}} U(1)_{Y}\right)$, where $\sqrt{\frac{5}{3}} U(1)_{Y}$ corresponds to the coupling $5 \alpha_{Y} / 3$ that unifies with $\alpha_{2}$ and $\alpha_{3}$ in the conventional MSSM.

\section{CONCLUSIONS}

We conclude with a few remarks contrasting the results obtained with those of the perturbative heterotic quasi-realistic models. The supersymmetry breaking in heterotic models has been extensively studied (see, e.g., [42,43] and references therein, and for recent studies [44].). One specific feature of heterotic models is that the tree level gauge couplings

\footnotetext{
${ }^{5}$ In the present case, the additional $U(1)$ factors are not broken at a high scale. $m_{Y}$ therefore refers to the diagonal $Y Y$ element of the gaugino mass matrix.
} 
are universal and depend only on one modulus $S$. Therefore, the gaugino condensation typically generates an effective superpotential that involves only one field, thus making the minimization of the supergravity potential a more intricate process. In addition, for a number of quasi-realistic models, while possessing an additional gauge sector, such sectors often were not confining (the beta functions were positive due to the additional matter). Further exploration involved the string threshold corrections that depend on toroidal moduli and allow for additional features of the supersymmetry breaking vacuum. In these examples the cosmological constant was in general large and negative and would have to be fixed by hand.

In contrast the supersymmetric models with intersecting D6 branes provide a framework with a confining gauge sector, where gaugino condensation can be addressed explicitly. We have demonstrated in an explicit example that the effective non-perturbative superpotential allows for the minimum of the supergravity potential in which supersymmetry is broken and the moduli (that determine the tree level gauge couplings at the string scale) are completely determined. Since the gauge couplings typically depend on more than one modulus, the minimization of the potential involves an interplay among all these moduli. The specific example has the property that the part of the potential that spontaneously breaks supersymmetry is much smaller than the $-3|W|^{2}$ term, resulting in a large and negative cosmological constant. We hope that other quasi-realistic models [28] with intersecting D6 branes may remedy this feature and possibly yield more realistic predictions, and plan to investigate dynamical supersymmetry breaking there.

\section{ACKNOWLEDGMENTS}

We would like to thank F. Cachazo, M. Douglas and expecially G. Shiu for useful discussions. M.C. would like to thank the New Center for Theoretical Physics at Rutgers University and the Institute for Advanced Study, Princeton, for hospitality and support during the course of this work. The research was supported in part by DOE grant DOEEY-76-02-3071, NATO the linkage grant No. 97061 (M.C.), and the Fay R. and Eugene L. Langberg Chair (M.C.). 


\section{REFERENCES}

[1] C. Angelantonj, M. Bianchi, G. Pradisi, A. Sagnotti and Ya.S. Stanev, Phys. Lett. B 385 (1996) 96.

[2] M. Berkooz and R.G. Leigh, Nucl. Phys. B 483 (1997) 187.

[3] Z. Kakushadze and G. Shiu, Phys. Rev. D 56 (1997) 3686; Nucl. Phys. B 520 (1998) 75;

Z. Kakushadze, Nucl. Phys. B 512 (1998) 221; Z. Kakushadze, G. Shiu and S. H. Tye, Nucl. Phys. B 533, 25 (1998).

[4] G. Zwart, Nucl. Phys. B 526 (1998) 378; D. O'Driscoll, hep-th/9801114;

[5] G. Shiu and S.-H.H. Tye, Phys. Rev. D58 (1998) 106007.

[6] J. Lykken, E. Poppitz and S. P. Trivedi, Nucl. Phys. B 543, 105 (1999).

[7] G. Aldazabal, A. Font, L.E. Ibáñez and G. Violero, Nucl. Phys. B 536 (1999) 29.

[8] Z. Kakushadze, Phys. Lett. B 434 (1998) 269; Phys. Rev. D 58 (1998) 101901; Nucl. Phys. B 535 (1998) 311.

[9] M. Cvetič, M. Plümacher and J. Wang, JHEP 0004 (2000) 004.

[10] M. Klein, R. Rabadán, JHEP 0010 (2000) 049.

[11] M. Cvetič, A. M. Uranga and J. Wang, Nucl. Phys. B 595, 63 (2001).

[12] G. Aldazabal, L. E. Ibanez, F. Quevedo and A. M. Uranga, hep-th/0005067.

[13] M. Berkooz, M. R. Douglas and R. G. Leigh, Nucl. Phys. B 480 (1996) 265.

[14] R. Blumenhagen, L. Görlich, B. Körs and D. Lüst, JHEP 0010 (2000) 006.

[15] G. Aldazabal, S. Franco, L. E. Ibáñez, R. Rabadán and A. M. Uranga, Journal of Mathematical Physics, vol. 42, number 7, p. 3103, hep-th/0011073; JHEP 0102 (2001) 047.

[16] R. Blumenhagen, B. Körs and D. Lüst, JHEP 0102 (2001) 030.

[17] L. E. Ibáñez, F. Marchesano and R. Rabadán, hep-th/0105155.

[18] C. Angelantonj, I. Antoniadis, E. Dudas and A. Sagnotti, Phys. Lett. B 489 (2000) 223.

[19] S. Förste, G. Honecker and R. Schreyer, Nucl. Phys. B 593 (2001) 127; JHEP 0106 (2001) 004.

[20] R. Blumenhagen, B. Körs and D. Lüst, T. Ott, Nucl. Phys. B616 (2001) 3.

[21] D. Cremades, L. E. Ibanez and F. Marchesano, Nucl. Phys. B 643, 93 (2002)

[22] D. Bailin, G. V. Kraniotis, and A. Love, Phys. Lett. B 530, 202 (2002); Phys. Lett. B 547, 43 (2002); hep-th/0210219; hep-th/0212112.

[23] C. Kokorelis, JHEP 0209, 029 (2002); JHEP 0208, 036 (2002); hep-th/0207234; JHEP 0211, 027 (2002); hep-th/0210200.

[24] M. Cvetič, G. Shiu and A. M. Uranga, Phys. Rev. Lett. 87, 201801 (2001).

[25] M. Cvetič, G. Shiu and A. M. Uranga, Nucl. Phys. B 615, 3 (2001).

[26] M. Cvetič , G. Shiu and A. M. Uranga, hep-th/0111179.

[27] R. Blumenhagen, L. Gorlich and T. Ott, hep-th/0211059.

[28] M. Cvetič and I. Papadimitriou, hep-th/0303197.

[29] M. Cvetič, I. Papadimitriou and G. Shiu, hep-th/0212177.

[30] M. Atiyah and E. Witten, hep-th/0107177.

[31] E. Witten, hep-th/0108165.

[32] B. Acharya and E. Witten, hep-th/0109152.

[33] E. Witten, hep-ph/0201018.

[34] T. Friedmann and E. Witten, hep-th/0211269.

[35] M. Cvetič, P. Langacker and G. Shiu, Phys. Rev. D 66, 066004 (2002). 
[36] M. Cvetič, P. Langacker and G. Shiu, Nucl. Phys. B 642, 139 (2002).

[37] G. Veneziano and S. Yankielowicz, Phys. Lett. B 113, 231 (1982).

[38] R. Dijkgraaf and C. Vafa, Nucl. Phys. B 644, 3 (2002).

[39] F. Cachazo, M. R. Douglas, N. Seiberg and E. Witten, hep-th/0211170.

[40] D. Cremades, L. E. Ibanez and F. Marchesano, "SUSY quivers, intersecting branes and the modest hierarchy problem," JHEP 0207, 009 (2002).

[41] D. Lüst and S. Stieberger, hep-th/0302221.

[42] J. A. Casas, Z. Lalak, C. Munoz and G. G. Ross, Nucl. Phys. B 347, 243 (1990).

[43] B. de Carlos, J. A. Casas and C. Munoz, Nucl. Phys. B 399, 623 (1993) .

[44] S. A. Abel, B. C. Allanach, F. Quevedo, L. Ibanez and M. Klein, JHEP 0012, 026 (2000). 